Maura Salvatori · Paolo M. Soardi

\title{
Multivariate compactly supported biorthogonal spline wavelets
}

Received: August 23, 2000; in final form: March 10, 2001

Published online: May 29, 2002 - (C) Springer-Verlag 2002

\begin{abstract}
We study biorthogonal bases of compactly supported wavelets constructed from box splines in $\mathbb{R}^{N}$ with any integer dilation factor. For a suitable class of box splines we write explicitly dual low-pass filters of arbitrarily high regularity and indicate how to construct the corresponding high-pass filters (primal and dual).
\end{abstract}

Mathematics Subject Classification (2000). 42C15, 42C40

\section{Introduction}

Biorthogonal bases of univariate compactly supported wavelets of arbitrarily high regularity were first constructed in 1992 by Cohen, Daubechies and Feauveau [7]. The innovation consisted in greater flexibilty in the filters' design and in avoiding the asymmetry and the numerical complexity of the coefficients of Daubechies' orthonormal wavelets. Since then, aside from the direct tensor product construction, there have been several extensions to higher dimensions and/or to general dilation matrices; see e.g. [4], [6], [9], [11], [12], [13], [16], [20], [21], [23]. As in the unidimensional case, special emphasis was put on compactly supported wavelets constructed from multivariate box splines, because spline functions are fairly well understood, to allow explicit computations and possess additional properties (see [2] for a general reference on box splines).

A general framework for the construction of biorthogonal wavelets is provided by multiresolution analyses. One starts with a refinable box spline $\varphi$ (with respect to an expanding matrix $S, S \mathbb{Z}^{N} \subset \mathbb{Z}^{N}$ ), generating a multiresolution analysis with a low-pass filter $m_{0}$ (i.e, the symbol of the refinement mask of $\varphi$ ); then one tries to construct a polynomial dual filter $\widetilde{m}_{0}$, which in turn defines a compactly supported dual distribution $\widetilde{\varphi}$ by means of an infinite product. If $\varphi$ and $\widetilde{\varphi}$ have Fourier transforms with enough decay at infinity and have mutually orthogonal translates, then the construction of biorthogonal wavelets amounts to solving a matrix extension problem. It was shown in [20], [16] that the Quillen-Suslin algorithm provides a constructive solution to this problem.

M. Salvatori: Dipartimento di Matematica dell’Università di Milano, via Saldini 50, 20133 Milano, Italy, e-mail: maura.salvatori@mat.unimi.it

P.M. Soardi: Dipartimento di Matematica e Applicazioni, Università di Milano-Bicocca, viale Sarca 202, 20126 Milano, Italy, e-mail: soardi@matapp.unimib. it 
Therefore, the concrete construction of a filter $\tilde{m}_{0}$ giving rise to a compactly supported dual function $\tilde{\varphi}$ of (arbitrarily) high regularity is a major issue in the theory of biorthogonal wavelets, and this problem was addressed in several of the above-mentioned papers. Generally speaking, there are only a few constructions available for dual pairs. In some cases a single pair is produced by explicit computation, but in many other cases it is possible to construct a whole family of filters which are dual to a given primal filter and generate a function $\widetilde{\varphi}$ of arbitrarily high regularity. As in the univariate case [7], one technique consists of constructing an appropriate filter, satisfying an interpolatory condition, which can be written as the product of a given filter and its dual. A general method for this construction appears in [16] and several applications are given. General as it may be, this method cannot guarantee arbitrarily high regularity in many important cases. Using a different method to construct the relevant polynomials, He and Lai explicitly produced the dual filters for a bivariate box spline in such a way that $\tilde{\varphi}$ has arbitrarily high regularity.

This paper was inspired by the above mentioned paper [13], and can be considered as an extension of the results and the methods contained therein. Namely, the He and Lai paper deals with bivariate box splines and the scaling matrix $2 I$, while we study biorthogonal compactly supported spline wavelets in any dimension $N$ with the scaling matrix $M \cdot I_{N}$, where $M \geq 2$ is an integer, $I_{N}$ is the identity matrix and the underlying lattice is $\mathbb{Z}^{N}$. While their construction is based on univariate Daubechies polynomials, our construction uses the multivariate Bernstein polynomials. We refer to [3] and [17] for the relation between Bernstein and Daubechies polynomials.

We assume that $\varphi=\varphi_{\Xi}$, is a box spline on $\mathbb{R}^{N}$, with full rank unimodular matrix $\Xi$ having integer entries. Such a spline is obviously refinable with respect to $M \cdot I_{N}$. In Section 2 we notice that, arguing along the lines of [21], there always exists a dual filter $\tilde{m}_{0}$ such that the corresponding $\widetilde{\varphi}$ is the convolution of a box spline and a compactly supported distribution. If $\widehat{\widetilde{\varphi}}$ has sufficient decay along the directions of $\Xi$, then we can prove that $\tilde{\varphi}$ and $\varphi$ have orthonormal translates. Therefore, the high-pass filters obtained by means of the Quillen-Suslin algorithm give rise to biorthogonal compactly supported wavelets.

Thus the problem arises of investigating which box splines (with unimodular $\Xi$ ) admit a dual polynomial filter $\widetilde{m}_{0}$ such that $\widehat{\widetilde{\varphi}}$ has the required decay. In Section 3 we prove that this is the case for box splines with Fourier transform of the type

$$
\widehat{\varphi}(\omega)=\prod_{k=1}^{N}\left(\frac{1-\exp \left(-i \omega_{k}\right)}{i \omega_{k}}\right)^{a_{k}} \prod_{k=2}^{N}\left(\frac{1-\exp \left(-i\left(\omega_{1}+\omega_{2}+\cdots+\omega_{k}\right)\right)}{i\left(\omega_{1}+\omega_{2}+\cdots+\omega_{k}\right)}\right)^{b_{k}}
$$

where $a_{k}>0$ and $b_{k} \geq 0$ are integers. Setting

$$
f(t)=\frac{1+e^{-i t}+e^{-i 2 t}+\cdots+e^{-i(M-1) t}}{M}
$$


we have that

$$
m_{0}(\omega)=\frac{\widehat{\varphi}(M \omega)}{\widehat{\varphi}(\omega)}=\prod_{k=1}^{N} f\left(\omega_{k}\right)^{a_{k}} \prod_{k=2}^{N} f\left(\omega_{1}+\omega_{2}+\cdots+\omega_{k}\right)^{b_{k}}
$$

For such splines, we will explicitly construct a dual filter $\tilde{m}_{0}$ of the form

$$
\tilde{m}_{0}(\omega)=\prod_{k=1}^{N} \bar{f}\left(\omega_{k}\right)^{\alpha_{k}-a_{k}} \prod_{k=2}^{N} \bar{f}\left(\omega_{1}+\omega_{2}+\cdots+\omega_{k}\right)^{\beta_{k}-b_{k}} \bar{r}(\omega),
$$

where $r(\omega)$ is a trigonometric polynomial. In the last section we will prove that the exponents $\alpha_{k}$ and $\beta_{k}$ can be chosen in such a way that $\tilde{\varphi}$, defined via its Fourier transform as

$$
\widehat{\widetilde{\varphi}}(\omega)=\prod_{j=1}^{\infty} \tilde{m}_{0}\left(M^{-j} \omega\right)
$$

has arbitrarily high regularity. It follows that every box spline of the form (1) gives rise to compactly supported biorthogonal wavelet bases and the dual wavelets can be constructed of arbitrarily high regularity.

\section{Biorthogonal spline wavelets}

We assume in this section that $\Xi=\left[\xi_{1}, \xi_{2}, \ldots, \xi_{K}\right]$, where $K \geq N$, is a full rank unimodular matrix (i.e., all the bases in $\Xi$ have determinant \pm 1 ) with integer entries. The box spline $\varphi_{\Xi}$ associated with $\Xi$ has Fourier transform

$$
\widehat{\varphi}_{\Xi}(\omega)=\prod_{j=1}^{K}\left(\frac{1-\exp \left(-i\left|\xi_{j}, \omega\right\rangle\right)}{i\left\langle\xi_{j}, \omega\right\rangle}\right) .
$$

In the following we will drop the subscript $\Xi$ and we will simply write $\varphi$ to denote this box spline. It is known that there exist constants $C_{1}, C_{2}>0$,

$$
C_{1} \leq \sum_{k \in \mathbb{Z}^{N}}|\widehat{\varphi}(\omega+2 \pi k)|^{2} \leq C_{2}
$$

and that $\varphi$ is the scaling function of a multiresolution analysis for any dilation factor $M \geq 2$ and translation lattice $\mathbb{Z}^{N}$ (see e.g. [15]). If $f$ is defined as in (2), then, as above, we set

$$
m_{0}(\omega)=\frac{\widehat{\varphi}(M \omega)}{\widehat{\varphi}(\omega)}=\prod_{j=1}^{K} f\left(\left\langle\xi_{j}, \omega\right\rangle\right)
$$

Now let $p(\omega)$ be any trigonometric polynomial. To $p(\omega)$ one associates a Laurent polynomial $P(z), z=\left(z_{1}, \ldots, z_{N}\right) \in(\mathbb{C} / 0)^{N}$, in such a way that $P\left(e^{i \omega}\right)=p(\omega)$, 
where we made $e^{i \omega}=\left(e^{i \omega_{1}}, \ldots, e^{i \omega_{N}}\right)$. In particular, we will denote by $P_{0}$ the Laurent polynomial such that $P_{0}\left(e^{i \omega}\right)=m_{0}(\omega)$. Clearly

$$
P_{0}(z)=\prod_{j=1}^{K} F\left(z^{\xi_{j}}\right)
$$

where we have adopted the usual multi-index notation and where

$$
F(t)=\frac{1+t^{-1}+t^{-2}+\cdots+t^{-(M-1)}}{M} .
$$

Throughout the paper we will use the following notation: we set $E=\frac{\mathbb{Z}^{N}}{M \mathbb{Z}^{N}}=$ $\{0,1, \ldots, M-1\}^{N}$ (with sum modulo $M$ ) and $\vartheta=\frac{2 \pi}{M}$. Furthermore, we set

$$
z \exp (i \vartheta \varepsilon)=\left(z_{1} \exp \left(i \vartheta \varepsilon_{1}\right), \ldots, z_{N} \exp \left(i \vartheta \varepsilon_{N}\right)\right)
$$

We start our analysis with the following proposition:

Proposition 1. Let $P_{0}$ be as in (7). Then, for every integer $c_{j}>0, j=1, \ldots, K$, there exists a Laurent polynomial $R(z)$ such that

$$
\widetilde{P}_{0}(z)=\prod_{j=1}^{K} F\left(z^{\xi_{j}}\right)^{c_{j}} R(z)
$$

satisfies the relation

$$
\sum_{\varepsilon \in E} P_{0}(z \exp (i \vartheta \varepsilon)) \widetilde{P}_{0}(z \exp (i \vartheta \varepsilon))=1 .
$$

Proof. Let

$$
Q(z)=\prod_{j=1}^{K} F\left(z^{\xi_{j}}\right)^{c_{j}+1} .
$$

Clearly, the matrix $\Xi^{\prime}$ in which the $j$-th column $\xi_{j}$ is repeated $c_{j}+1$ times is still unimodular. Then, it was proved in [21, Proposition 1.11] (for $M=2$, but the proof is exactly the same for general $M$ ) that the set of polynomials

$$
\{Q(z \exp (i \vartheta \varepsilon))\}_{\varepsilon \in E}
$$

do not have common zeros. Therefore, by Hilbert Nullstellensatz (exactly as in [9, Lemma 3.2.1], or in [21, Proposition 1.11]), there exists a Laurent polynomial $R$ such that

$$
\sum_{\varepsilon \in E} Q(z \exp (i \vartheta \varepsilon)) R(z \exp (i \vartheta \varepsilon))=1 .
$$

This immediately implies (9). 
The trigonometric polynomial

$$
\widetilde{m}_{0}(\omega)={\widetilde{P_{0}}}_{0}\left(e^{i \omega}\right)=\prod_{j=1}^{K} \bar{f}\left(\left\langle\xi_{j}, \omega\right\rangle\right)^{c_{j}} \bar{r}(\omega),
$$

where $r(\omega)=R\left(e^{i \omega}\right)$, is the dual filter of $m_{0}$ and satisfies (15) below (with $t=s=0)$. Now we will proceed to construct the wavelet filters.

First we notice that, if $P$ and $\widetilde{P}$ are Laurent polynomials represented by means of polyphase components in the forms

$$
P=M^{-N / 2} \sum_{\varepsilon \in E} P_{\varepsilon}\left(z^{M}\right) z^{\varepsilon} \text { and } \widetilde{P}=M^{-N / 2} \sum_{\varepsilon \in E} \widetilde{P}_{\varepsilon}\left(z^{M}\right) z^{-\varepsilon}
$$

(where $\left.z^{M}=\left(z_{1}^{M}, \ldots, z_{N}^{M}\right)\right)$, then clearly

$$
\sum_{\varepsilon \in E} P(z \exp (i \vartheta \varepsilon)) \widetilde{P}(z \exp (i \vartheta \varepsilon))=\sum_{\varepsilon \in E} P_{\varepsilon}\left(z^{M}\right) \widetilde{P}_{\varepsilon}\left(z^{M}\right) .
$$

In particular, we will denote by $P_{0, \varepsilon}$ and $\widetilde{P}_{0, \varepsilon}$ the polyphase components $(11)$ of the polynomials $P_{0}$ and $\widetilde{P}_{0}$ of Proposition 1 . The following extension result is proved in [20] (see also [16]), but, for the sake of completeness, we will give a short account of the proof.

Proposition 2. Let $P_{0}$ and $\widetilde{P}_{0}$ satisfy (9), and denote by $P_{0, \varepsilon}$ and $\widetilde{P}_{0, \varepsilon}$ their polyphase components (11). Then there exist $M^{N} \times M^{N}$ matrices $\left[P_{s, \varepsilon}\right]$ and $\left[\widetilde{P}_{s, \varepsilon}\right]$, whose entries are Laurent polynomials and whose first rows are $\left[P_{0, \varepsilon}\right]$ and $\left[\widetilde{P}_{0, \varepsilon}\right]$, such that $\left[P_{s, \varepsilon}\right] \cdot\left[\widetilde{P}_{s, \varepsilon}\right]^{T}=I$.

Proof. We use the same notation to denote the polynomials $P_{0}$ and $\widetilde{P}_{0}$ and the vectors $\left[P_{0, \varepsilon}\right]$ and $\left[\widetilde{P}_{0, \varepsilon}\right]$. Taking into account (9) and (12) we can apply the Quillen-Suslin algorithm [18] to the vectors $P_{0}$ and $\widetilde{P}_{0}$. Then there are $M^{N}-1$ vectors $Q_{s}$, having polynomial components, such that the matrix with first row $P_{0}$ and $s$-th row $Q_{s}$ is not singular on $(\mathbb{C} / 0)^{N}$. Set

$$
P_{s}=Q_{s}-\left(Q_{s} \cdot \widetilde{P}_{0}^{T}\right) P_{0}
$$

Then $P_{s}=\left[P_{s, \varepsilon}\right]$ is orthogonal to $\widetilde{P}_{0}$. The matrix whose rows are $P_{0}$ and $P_{s}$ is still nonsingular, since the equation $\lambda_{0} P_{0}+\sum_{s} \lambda_{s} P_{s}=0$ implies

$$
\left(\lambda_{0}-\sum_{s=1}^{M^{N}-1}\left(Q_{s} \cdot \widetilde{P}_{0}^{T}\right)\right) P_{0}+\sum_{s=1}^{M^{N}-1} \lambda_{s} Q_{s}=0
$$

hence $\lambda_{s}=0$ for $s=0,1, \ldots, M^{N}-1$. Thus its determinant is a Laurent monomial, and the elements of its inverse $\left[H_{s, \varepsilon}\right]^{T}$ are Laurent polynomials. The first row in this matrix is necessarily $\widetilde{P}_{0}$. Thus, letting $\widetilde{P}_{s, \varepsilon}=H_{s, \varepsilon}$ for $s=1, \ldots, M^{N}-1$, we have the thesis. 
Remark 1. The above proof is based on Theorem 1.1 in [18], whose proof is algorithmic, and each step of the algorithm can be implemented by means of computer algebra systems.

Let $P_{s, \varepsilon}$ and $\widetilde{P}_{s, \varepsilon}$ be as in Proposition 2. We set

$$
\begin{aligned}
& m_{s}(\omega)=M^{-N / 2} \sum_{\varepsilon \in E} P_{s, \varepsilon}\left(e^{i M \omega}\right) e^{i\langle\varepsilon, \omega\rangle} \\
& \tilde{m}_{S}(\omega)=M^{-N / 2} \sum_{\varepsilon \in E} \widetilde{P_{s, \varepsilon}}\left(e^{i M \omega}\right) e^{i\langle\varepsilon, \omega\rangle} .
\end{aligned}
$$

Taking into account (12) and Propositions 1 and 2, we see that the filters just defined satisfy, for all $\omega \in \mathbb{R}^{N}$, the system

$$
\sum_{\varepsilon \in E} m_{s}(\omega+\vartheta \varepsilon) \overline{\widetilde{m}_{t}(\omega+\vartheta \varepsilon)}=\delta_{s, t}, \quad t, s=0,1, \ldots, M^{N}-1,
$$

or, equivalently,

$$
\sum_{s=0}^{M^{N}-1} m_{s}(\omega) \overline{\widetilde{m}_{s}(\omega+\vartheta \varepsilon)}=\delta_{0, \varepsilon}, \quad \varepsilon \in E .
$$

In order to interpret these filters as arising from dual multiresolution analyses and biorthogonal wavelets, we need $m_{0}$ and $\tilde{m}_{0}$ to be low-pass, and $m_{s}$ and $\tilde{m}_{s}$, for $s=1, \ldots, M^{N}-1$, to be high-pass, in the sense made precise by the following lemma:

Lemma 1. Let $m_{0}$ be defined as in (6) and $\tilde{m}_{0}$ as in (10). Moreover, let $m_{s}$ and $\tilde{m}_{s}$ be defined by means of Proposition 2 and (13), (14) for $s \geq 1$. Then,

$$
\begin{aligned}
\tilde{m}_{0}(0) & =m_{0}(0)=1, \\
\tilde{m}_{0}(\vartheta \varepsilon) & =m_{0}(\vartheta \varepsilon)=0 \text { for every } \varepsilon \neq 0, \\
\tilde{m}_{s}(0) & =m_{s}(0)=0, \text { for every } s=1, \ldots, M^{N}-1 .
\end{aligned}
$$

Proof. Equation $m_{0}(0)=1$ is obvious. To prove (18) observe that if, say, $\xi_{j_{1}}, \ldots, \xi_{j_{N}}$ are columns of $\Xi$ forming a basis, then, by the unimodularity assumption, the matrix $\left[\xi_{j_{1}}, \ldots, \xi_{j_{N}}\right]$ can be transformed into the unit matrix by means of a change of variables with integer coefficients and determinant \pm 1 . It follows that for every $\varepsilon \in E$ there exists a $j_{k}$ such that $f\left(\left|\xi_{j_{k}}, \vartheta \varepsilon\right\rangle\right)=0$, and hence (18). Now, using (15) with $s=t=0$ and $\omega=0$, we also get $\widetilde{m}_{0}(0)=1$. Thus (17) and (18) hold. Now, we make $\omega=0$ in system (16), and we express the $m_{s}(0)$ by means of $\overline{\widetilde{m}_{s}(\vartheta \varepsilon)}$. We see immediately that any minor corresponding to $s>0$ and $\varepsilon=0$ must vanish, since it contains the row $\left\{\tilde{m}_{0}(\vartheta \varepsilon)\right\}_{\varepsilon \neq 0}$. Thus $m_{s}(0)=0$. The analogous statement for $\tilde{m}_{s}(0)$ follows, exchanging the roles of $m_{s}$ and $\tilde{m}_{s}$. 
Define $\widehat{\widetilde{\varphi}}(\omega)$ as in (5), with $\widetilde{m}_{0}$ as in (10). Clearly $\widetilde{\varphi}$ is a compactly supported distribution with $\widehat{\widetilde{\varphi}}(0)=1$, which is the convolution of the box spline with Fourier transform

$$
\prod_{j=1}^{K}\left(\frac{1-\exp \left(-i\left\langle\xi_{j}, \omega\right\rangle\right)}{i\left\langle\xi_{j}, \omega\right\rangle}\right)^{c_{j}}
$$

and the compactly supported distribution with Fourier transform

$$
\prod_{j=1}^{\infty} \bar{r}\left(M^{-j} \omega\right)
$$

where $r(\omega)=R\left(e^{i \omega}\right)$. We set, for every $s=1, \ldots, M^{N}-1$,

$$
\widehat{\psi}_{s}(M \omega)=m_{s}(\omega) \widehat{\varphi}(\omega), \quad \widehat{\widetilde{\psi}}_{s}(M \omega)=\widetilde{m}_{s}(\omega) \widehat{\widetilde{\varphi}}(\omega) .
$$

Clearly we have

$$
|\widehat{\varphi}(\omega)| \leq C \prod_{j=1}^{K}\left(1+\left|\left\langle\xi_{j}, \omega\right\rangle\right|\right)^{-1},
$$

where $C$ is a constant, and $\widehat{\psi}_{s}$ has the same decay. On the other hand, the decay of (20) at infinity of is bounded from above by $\prod_{j=1}^{K}\left(1+\left|\left\langle\xi_{j}, \omega\right\rangle\right|\right)^{-c_{j}}$.

In the rest of this section we will assume that the Fourier transform (21) does not increase too much at infinity. Namely we assume that

$$
\widetilde{\varphi} \in L^{2}\left(\mathbb{R}^{N}\right)
$$

and that there exist $\sigma>0$ and $C>0$ such that, for every sufficiently large $n$, one has, for all $\omega \in \mathbb{R}^{N}$,

$$
\prod_{h=1}^{n}\left|r\left(M^{-h} \omega\right)\right| \leq C \prod_{j=1}^{K}\left(1+\left|\left\langle\xi_{j}, \omega\right\rangle\right|\right)^{c_{j}-\sigma} .
$$

Obviously, since $\Xi$ is full rank, if (24) holds with $\sigma>1 / 2$ then (23) is satisfied as well.

Remark 2. Assumptions (23), (24) seem to be hard to verify. Nevertheless, in the following sections we will show that for a remarkable class of box splines we can prove much better estimates (Theorem 3 below), making it possible to construct the square integrable $\tilde{\varphi}$ of arbitrarily high regularity.

Let $\psi$ and $\widetilde{\psi}$ be as in (22). We set, for every $j \in \mathbb{Z}, k \in \mathbb{Z}^{N}$ and $s=$ $1, \ldots, M^{N}-1$,

$$
\psi_{j, k, s}(x)=M^{j / 2} \psi_{s}\left(M^{j} x-k\right), \quad \widetilde{\psi}_{j, k, s}(x)=M^{j / 2} \widetilde{\psi}_{s}\left(M^{j} x-k\right) .
$$


Theorem 1. Let $\varphi$ be any box spline in $\mathbb{R}^{N}$ with full rank unimodular matrix $\Xi$ having integer entries. Let $\widehat{\widetilde{\varphi}}(\omega)$, as in (5), with $\widetilde{m}_{0}$ as in (8) and (10). Assume that (23) and (24) hold. Then the families $\left\{\psi_{j, k, s}\right\}$ and $\left\{\widetilde{\psi}_{j, k, s}\right\}$ in (25) are biorthogonal bases of compactly supported wavelets in $L^{2}\left(\mathbb{R}^{N}\right)$.

Proof. By (24), (17) and (19) we may apply Theorem 2.11 of Chui and Li in [4]. Thus we conclude that both systems $\left\{\psi_{j, k, s}\right\}$ and $\left\{\widetilde{\psi}_{j, k, s}\right\}$ are Bessel systems i.e., for every function $g \in L^{2}\left(\mathbb{R}^{N}\right)$,

$$
\begin{aligned}
& \sum_{j, k, s}\left|\left\langle g, \psi_{j, k, s}\right\rangle\right|^{2} \leq C\|g\|_{2}^{2} \\
& \sum_{j, k, s}\left|\left\langle g, \widetilde{\psi}_{j, k, s}\right\rangle\right|^{2} \leq C\|g\|_{2}^{2}
\end{aligned}
$$

for some constant $C$. Then we may apply Ron and Shen's theorem 3.9 in [22] (or we can repeat the proof in [7, pp. 500-506]) to conclude that the two families are fundamental frames which are dual to each other. Therefore, to obtain the thesis it will be sufficient to prove that the families are biorthogonal i.e.,

$$
\left\langle\psi_{j, k, s}, \widetilde{\psi}_{i, h, t}\right\rangle=\delta_{j, i} \delta_{k, h} \delta_{s, t}
$$

To this end, by [4, Theorem 2.2] (or repeating the arguments in [7, Lemma 3.7]) we have only to prove the analogous relation for the scaling functions i.e.,

$$
\int_{\mathbb{R}^{N}} \varphi(x) \overline{\widetilde{\varphi}(x-k)} d x=\delta_{0, k}
$$

We will prove (26) by arguing along the lines of [7]. Let us denote by $\Omega$ the fundamental domain for the box spline

$$
\widehat{\varphi^{\sharp}}(\omega)=\prod_{j=1}^{K}\left(\frac{1-\exp \left(-i\left\langle\xi_{j}, \omega\right\rangle\right)}{i\left\langle\xi_{j}, \omega\right\rangle}\right)^{c_{j}+1} .
$$

We recall [2, p. 106] that $\Omega$ is defined as

$$
\Omega=\left\{\omega:\left|\widehat{\varphi^{\sharp}}(\omega+2 \pi k)\right|<\left|\widehat{\varphi^{\sharp}}(\omega)\right| \text { for all } k \in \mathbb{Z}^{N}, k \neq 0\right\} .
$$

It turns out that meas $(\Omega)=(2 \pi)^{N}$, the translates $\Omega+2 \pi k$ are pairwise disjoint and tessellate $\mathbb{R}^{N}$ up to a set of measure 0 . Moreover, $\bar{\Omega}$ is compact and, since $\varphi^{\sharp}$ has unimodular matrix,

$$
\left|\widehat{\varphi^{\sharp}}(\omega)\right| \geq \kappa>0 \text {, for every } \omega \in \bar{\Omega} .
$$

As a consequence we obtain

$$
\prod_{j=1}^{K}\left|\sin \left(\left\langle\xi_{j}, \omega / 2\right\rangle\right)\right|^{c_{j}+1} \geq \kappa \prod_{j=1}^{K}\left|\left\langle\xi_{j}, \omega\right\rangle\right|^{c_{j}+1}, \quad \text { for every } \omega \in \bar{\Omega} .
$$


Now define $\widehat{\varphi}_{0}(\omega)=\widehat{\widetilde{\varphi}}_{0}(\omega)=\chi(\omega)$, where $\chi$ is the characteristic function of $\Omega$, and, for $n=1,2, \ldots$

$$
\begin{aligned}
& \widehat{\varphi}_{n}(\omega)=\chi\left(M^{-n} \omega\right) \prod_{h=1}^{n} m_{0}\left(M^{-h} \omega\right) \\
& \widehat{\widetilde{\varphi}}_{n}(\omega)=\chi\left(M^{-n} \omega\right) \prod_{h=1}^{n} \tilde{m}_{0}\left(M^{-h} \omega\right) .
\end{aligned}
$$

Since, for any periodic function $g,(2 \pi)^{-N} \int_{\Omega} g d \omega=\int_{\mathbb{T}^{N}} g d \omega$ (where $\mathbb{T}^{N}$ is the $N$-dimensional torus), a standard argument based on (15) with $s=t=0$ (cf. [7, p. 528]) shows that

$$
\int_{\mathbb{R}^{N}} \varphi_{n}(x) \overline{\widetilde{\varphi}_{n}(x-k)} d x=(2 \pi)^{-N} \int_{\mathbb{R}^{N}} \widehat{\varphi}_{n}(\omega) \overline{\widehat{\widetilde{\varphi}}_{n}(\omega)} \exp (i k \omega) d \omega=\delta_{0, k} .
$$

Now we look for an integrable majorant in order to apply the Lebesgue theorem. We have that $M|f(t)|=|1-\exp (-i M t)||1-\exp (-i t)|^{-1}$. Hence, by (24) and (27), we see that for some constant $C$

$$
\begin{aligned}
\left|\widehat{\varphi}_{n}(\omega) \widehat{\tilde{\varphi}}_{n}(\omega)\right| & =\chi\left(M^{-n} \omega\right) \prod_{j=1}^{K}\left|\frac{\sin \left(\left\langle\xi_{j}, \omega / 2\right\rangle\right)}{M^{n} \sin \left(\left\langle\xi_{j}, M^{-n} \omega / 2\right\rangle\right)}\right|^{c_{j}+1} \prod_{j=1}^{n}\left|r\left(M^{-j} \omega\right)\right| \\
& \leq \kappa^{-1} \prod_{j=1}^{K}\left|\frac{\sin \left(\left\langle\xi_{j}, \omega / 2\right\rangle\right)}{\left\langle\xi_{j}, \omega\right\rangle}\right|^{c_{j}+1} \prod_{j=1}^{n}\left|r\left(M^{-j} \omega\right)\right| \\
& \leq C \prod_{j=1}^{K}\left(1+\left|\left\langle\xi_{j}, \omega\right\rangle\right|\right)^{-1-\sigma} .
\end{aligned}
$$

As $\varphi_{n} \rightarrow \varphi$ and $\widehat{\varphi}_{n} \rightarrow \widehat{\varphi}$ pointwise, (26) follows from the Lebesgue theorem.

\section{Construction of the dual filter}

Proposition 1 provides an abstract construction of the dual filter, but does not give information on the form of the "residual filter" $r$, nor on the regularity of the dual scaling function. On the other hand, Theorem 1 proves that $\left\{\psi_{j, k, s}\right\}$ and $\left\{\widetilde{\psi}_{j, k, s}\right\}$ in (25) are biorthogonal bases provided that a decay condition on the Fourier transform side is satisfied. In this section we restrict the scope of our investigation, considering only spline filters of the form (3). For such filters we construct dual filters $\widetilde{m}_{0}$ of the form (4) by explicitly giving the expression of the trigonometric polynomial $r$. In the next section we will show that for such filters, the assumptions of Theorem 1 are satisfied, thus obtaining biorthogonal compactly supported wavelets. At the same time, we will show that the resulting dual scaling function $\widetilde{\varphi}$ can be constructed of arbitrarily high regularity. 
Our starting point are the polynomials studied in [24] and [23] in the context of univariate compactly supported $M$-band wavelets (also cf. [16]). We set

$$
\begin{aligned}
& S_{M}=\left\{\left(s_{0}, \ldots, s_{M-1}\right): s_{i} \geq 0, s_{0}+\cdots+s_{M-1}=1\right\} \\
& T_{M}=\left\{\left(s_{0}, \ldots, s_{M-1}\right) \in S_{M}: s_{0} \geq 1 / M\right\}
\end{aligned}
$$

and we denote by $\chi_{M}$ the characteristic function of $T_{M}$. We define the function $g$ on $S_{M}$ as

$$
\begin{aligned}
& g\left(s_{0}, \ldots, s_{M-1}\right)= \\
& \quad \frac{\chi_{M}\left(s_{0}, \ldots, s_{M-1}\right)}{\chi_{M}\left(s_{0}, \ldots, s_{M-1}\right)+\chi_{M}\left(s_{1}, s_{2}, \ldots, s_{0}\right)+\cdots+\chi_{M}\left(s_{M-1}, s_{0} \ldots, s_{M-2}\right)} .
\end{aligned}
$$

Note that the value of $g$ at $\left(s_{0}, \ldots, s_{M-1}\right) \in T_{M}$ is the reciprocal of the number of variables $s_{i}$ not smaller than $1 / M$. We observe the following properties of $g$ on $S_{M}$ :

$$
\begin{gathered}
g\left(s_{0}, \ldots, s_{M-1}\right)+g\left(s_{M-1}, s_{0}, \ldots, s_{M-2}\right)+\cdots+g\left(s_{1}, s_{2}, \ldots, s_{0}\right)=1 \\
\frac{1}{M} \leq g\left(s_{0}, \ldots, s_{M-1}\right) \leq 1 \text { if } s_{0} \geq 1 / M, \\
g\left(s_{0}, \ldots, s_{M-1}\right)=0 \text { if } s_{0}<1 / M .
\end{gathered}
$$

For every integer $k \geq 2$, we define the polynomials $Q_{k}$ as

$$
\begin{aligned}
& Q_{k}\left(y_{0}, \ldots, y_{M-1}\right)= \\
& \sum_{|j|=M(k-1)+1}\left(\begin{array}{c}
M(k-1)+1 \\
j
\end{array}\right) g\left(\frac{j_{0}}{M(k-1)+1}, \ldots, \frac{j_{M-1}}{M(k-1)+1}\right) y_{0}^{j_{0}-k} y_{1}^{j_{1}} \cdots y_{M-1}^{j_{M-1}}
\end{aligned}
$$

(where $j=\left(j_{0}, \ldots, j_{M-1}\right),|j|$ is the length of the multindex $j$ and $j !=$ $\left.j_{0} ! \cdots j_{M-1} !\right)$.

Taking (28) and (29) into account it is not difficult to check that

$$
\begin{aligned}
\left(y_{0}+\cdots\right. & \left.+y_{M-1}\right)^{M(k-1)+1}=y_{0}^{k} Q_{k}\left(y_{0}, \ldots, y_{M-1}\right) \\
& +y_{1}^{k} Q_{k}\left(y_{1}, y_{2}, \ldots, y_{0}\right)+\cdots+y_{M-1}^{k} Q_{k}\left(y_{M-1}, y_{0}, \ldots, y_{M-2}\right) .
\end{aligned}
$$

Actually, a moment's reflection shows that for $y_{0}+\cdots+y_{M-1}=1$ the polynomial $y_{0}^{k} Q_{k}\left(y_{0}, \ldots, y_{M-1}\right)$ is nothing but the Bernstein polynomial of order $M(k-1)+1$ of the function $g$ on the $M$-dimensional simplex [19, p. 51]. In the case $M=2$ we have

$$
\begin{aligned}
Q_{k}(1-y, y) & =\sum_{j=k}^{2 k-1}\left(\begin{array}{c}
2 k-1 \\
j
\end{array}\right)(1-y)^{j-k} y^{2 k-1-j} \\
& =\sum_{j=0}^{k-1}\left(\begin{array}{c}
k-1+j \\
j
\end{array}\right) y^{j}
\end{aligned}
$$


so that the polynomial $P_{k}(y)=Q_{k}(1-y, y)$ is the well-known Daubechies polynomial [10]. The relation of the Daubechies polynomials with the Bernstein polynomials was pointed out in [3] and [17].

Let $f$ be as in (2), and let, as in the foregoing section, $\vartheta=2 \pi / M$. Let us set

$$
y_{0}(t, u)=f(t) f(u)=M^{-2} \sum_{a, b=0}^{M-1} \exp [-i(a t+b u)],
$$

and, for every $\ell=1, \ldots, M-1$,

$$
y_{\ell}(t, u)=y_{0}(t+\ell \vartheta, u+(M-\ell) \vartheta)=f(t+\ell \vartheta) f(u+(M-\ell) \vartheta) .
$$

Finally, we set

$$
A_{k}(t, u)=Q_{k}\left(y_{0}(t, u), \ldots, y_{M-1}(t, u)\right),
$$

where $Q_{k}$ is as in (30). Using (33)-(34), the $A_{k}$ can be written explicitly as

$$
\begin{aligned}
A_{k}(t, u)= & \sum_{|j|=M(k-1)+1}\left(\begin{array}{c}
M(k-1)+1 \\
j
\end{array}\right) g\left(\frac{j_{0}}{M(k-1)+1}, \ldots, \frac{j_{M-1}}{M(k-1)+1}\right) \\
& \times\left(\frac{e^{-i(M-1)(t+u) / 2}}{M^{2}} \frac{\sin \frac{M t}{2}}{\sin \frac{t}{2}} \frac{\sin \frac{M u}{2}}{\sin \frac{u}{2}}\right)^{j_{0}-k} \\
& \times \prod_{\ell=1}^{M-1}\left(\frac{e^{-i(M-1)(t+u) / 2}}{M^{2}} \frac{\sin \left(\frac{M t}{2}\right)}{\sin \left(\frac{t}{2}+\frac{\ell \pi}{M}\right)} \frac{\sin \left(\frac{M u}{2}\right)}{\sin \left(\frac{u}{2}-\frac{\ell \pi}{M}\right)}\right)^{j_{\ell}},
\end{aligned}
$$

since $f(t)=M^{-1} e^{-i(M-1) t / 2} \sin \frac{M t}{2} / \sin \frac{t}{2}$.

We have the following lemma:

Lemma 2. With notation as above, we have, for every $k \geq 2$,

$\sum_{\ell=0}^{M}[f(t+\ell \vartheta) f(u+(M-\ell) \vartheta)]^{k} A_{k}(t+\ell \vartheta, u+(M-\ell) \vartheta)=[f(t+u)]^{M(k-1)+1}$.

Proof. The lemma is a consequence of (31). For every $t, u \in \mathbb{R}$ and $\ell=1, \ldots$, $M-1$ we have

$$
M^{2} f(t+\ell \vartheta) f(u+(M-\ell) \vartheta)=\sum_{a, b=0}^{M-1} \exp [-i(a t+a \ell \vartheta+b u+b(M-\ell) \vartheta)]
$$

Summing up all these relations we obtain

$$
\begin{aligned}
M^{2} \sum_{\ell=0}^{M-1} f(t+\ell \vartheta) & f(u+(M-\ell) \vartheta) \\
& \left.=\sum_{a, b=0}^{M-1} \exp [-i(a t+b u)] \sum_{\ell=0}^{M-1} \exp [-i \vartheta(a-b) \ell)\right] \\
& =M \sum_{a=0}^{M-1} \exp [-i a(t+u)]=M^{2} f(t+u) .
\end{aligned}
$$


Now, since $y_{h}(t+\ell \vartheta, u+(M-\ell) \vartheta)=y_{\ell+h}(t, u)$ (where the sum $\ell+h$ in the subscript is $\bmod M$ ), we have

$$
A_{k}(t+\ell \vartheta, u+(M-\ell) \vartheta)=Q_{k}\left(y_{\ell}(t, u), y_{\ell+1}(t, u), \ldots, y_{\ell-1}(t, u)\right) .
$$

Thus the thesis of the lemma follows from (31) and (36).

Let us introduce some more notation. First we have that

$$
f(t)^{2}=\frac{1}{M^{2}} \frac{\sin ^{2}\left(\frac{M t}{2}\right)}{\sin ^{2}\left(\frac{t}{2}\right)} \exp [-i(M-1) t]=|f(t)|^{2} \exp [-i(M-1) t],
$$

and that $\sum_{j=0}^{M-1}|f(t+j \vartheta)|^{2}=1$. Then we set

$$
\begin{aligned}
B_{k}(t) & =\exp [i k(M-1) t] Q_{k}\left(|f(t)|^{2}, \ldots,|f(t+(M-1) \vartheta)|^{2}\right) \\
& =e^{i(M-1) k t} \sum_{|j|=M(k-1)+1}\left(\begin{array}{c}
M(k-1)+1 \\
j
\end{array}\right) g\left(\frac{j_{0}}{M(k-1)+1}, \ldots, \frac{j_{M-1}}{M(k-1)+1}\right) \\
& \times\left(\frac{\sin ^{2}\left(\frac{M t}{2}\right)}{M^{2} \sin ^{2}\left(\frac{t}{2}\right)}\right)^{j_{0}-k} \prod_{\ell=1}^{M-1}\left(\frac{\sin ^{2}\left(\frac{M t}{2}\right)}{M^{2} \sin ^{2}\left(\frac{t}{2}+\frac{\ell \pi}{M}\right)}\right)^{j_{\ell}} .
\end{aligned}
$$

Using (31) and arguing much in the same way as in the preceding lemma, one proves easily the following result:

Lemma 3. With notation as above, we have, for every $k \geq 2$,

$$
\sum_{\ell=0}^{M-1} f(t+\ell \vartheta)^{2 k} B_{k}(t+\ell \vartheta)=1
$$

Remark 3. If $M=2$, then $B_{k}(t)=\exp (i k t) P_{k}\left(\sin ^{2} t / 2\right)$, where $P_{k}$ is as in (32). Hence, the filters $f(t)^{2 k} B_{k}(t)$ can be considered as an extension to the case $M>2$ of Daubechies filters.

Now we can state the main result of this section.

Theorem 2. Let $N>1$ and

$$
m_{0}(\omega)=\prod_{k=1}^{N} f\left(\omega_{k}\right)^{a_{k}} \prod_{k=2}^{N} f\left(\omega_{1}+\cdots+\omega_{k}\right)^{b_{k}}
$$

where $f$ is as in (2), $a_{k}>0$ and $b_{k} \geq 0$ are integers. Set

$$
\tilde{m}_{0}(\omega)=\prod_{k=1}^{N} \bar{f}\left(\omega_{k}\right)^{\alpha_{k}-a_{k}} \prod_{k=2}^{N} \bar{f}\left(\omega_{1}+\cdots+\omega_{k}\right)^{\beta_{k}-b_{k}} \bar{r}(\omega),
$$

with

$$
r(\omega)=B_{\alpha_{N+1}}\left(\omega_{1}+\cdots+\omega_{N}\right) \prod_{k=1}^{N-1} A_{\alpha_{k+1}}\left(\omega_{1}+\cdots+\omega_{k}, \omega_{k+1}\right),
$$


where $\alpha_{k}, \beta_{k}>0$ are integers and the trigonometric polynomials $A$ and $B$ are defined as in (35) and (37), respectively. Then for every $\omega \in \mathbb{R}^{N}$,

$$
\sum_{\varepsilon \in E} m_{0}(\omega+\vartheta \varepsilon) \overline{\widetilde{m}_{0}(\omega+\vartheta \varepsilon)}=1,
$$

provided that

$$
\left\{\begin{array}{l}
\alpha_{1}=\alpha_{2} \\
\alpha_{k+1}=\beta_{k}+M\left(\alpha_{k}-1\right)+1 \\
2 \alpha_{N+1}=\beta_{N}+M\left(\alpha_{N}-1\right)+1
\end{array} \text { if } 2 \leq k \leq N-1\right.
$$

Proof. Let

$$
\begin{aligned}
h(\omega) & =m_{0}(\omega) \overline{\widetilde{m}_{0}(\omega)} \\
& =\prod_{k=1}^{N} f\left(\omega_{k}\right)^{\alpha_{k}} \prod_{k=2}^{N} f\left(\omega_{1}+\cdots+\omega_{k}\right)^{\beta_{k}} \\
& \times B_{\alpha_{N+1}}\left(\omega_{1}+\cdots+\omega_{N}\right) \prod_{k=1}^{N-1} A_{\alpha_{k+1}}\left(\omega_{1} \cdots+\omega_{k}, \omega_{k+1}\right) .
\end{aligned}
$$

Then

$$
h(\omega)=D(\omega) \prod_{k=1}^{N-1} C_{k}(\omega)
$$

where, for every $k=1, \ldots, N-1$,

$$
C_{k}(\omega)=f\left(\omega_{1}+\cdots+\omega_{k}\right)^{\beta_{k}} f\left(\omega_{k+1}\right)^{\alpha_{k+1}} A_{\alpha_{k+1}}\left(\omega_{1}+\cdots+\omega_{k}, \omega_{k+1}\right)
$$

and

$$
D(\omega)=f\left(\omega_{1}+\cdots+\omega_{N}\right)^{\beta_{N}} B_{\alpha_{N+1}}\left(\omega_{1}+\cdots+\omega_{N}\right) .
$$

Here we set $\beta_{1}=\alpha_{1}$. For every $k \geq 2$ we consider the elements $\varepsilon^{(k)}$ of $E$ with the first coordinate equal to 1 , the $k$-th equal to $M-1$ and the remaining coordinates equal to 0 , i.e.

$$
\varepsilon^{(k)}=(1,0, \ldots, M-1,0, \ldots, 0) .
$$

Set

$$
\begin{aligned}
E_{2} & =\left\{\varepsilon \in E: \varepsilon=j \varepsilon^{(2)}, j=0, \ldots, M-1\right\} \\
& =\{\varepsilon \in E: \varepsilon=(j, M-j, 0, \ldots, 0), j=0, \ldots, M-1\} .
\end{aligned}
$$

Clearly $\left|E_{2}\right|=M$, where $|\cdot|$ denotes the cardinality. We have

$$
\sum_{\varepsilon \in E_{2}} h(\omega+\vartheta \varepsilon)=\sum_{\varepsilon \in E_{2}} C_{1}(\omega+\vartheta \varepsilon) \prod_{k=2}^{N-1} C_{k}(\omega) D(\omega)
$$


and, since $\alpha_{1}=\alpha_{2}$, by Lemma 2 we obtain

$$
\begin{aligned}
& \sum_{\varepsilon \in E_{2}} C_{1}(\omega+\vartheta \varepsilon) \\
& =\sum_{j=0}^{M-1} f\left(\omega_{1}+j \vartheta\right)^{\alpha_{1}} f\left(\omega_{2}+(M-j) \vartheta\right)^{\alpha_{2}} A_{\alpha_{2}}\left(\omega_{1}+j \vartheta, \omega_{2}+(M-j) \vartheta\right) \\
& =f\left(\omega_{1}+\omega_{2}\right)^{M\left(\alpha_{2}-1\right)+1} .
\end{aligned}
$$

Using the relation $\alpha_{3}=\beta_{2}+M\left(\alpha_{2}-1\right)+1$ we can also write

$$
\sum_{\varepsilon \in E_{2}} h(\omega+\vartheta \varepsilon)=f\left(\omega_{1}+\omega_{2}\right)^{\alpha_{3}} f\left(\omega_{3}\right)^{\alpha_{3}} A_{\alpha_{3}}\left(\omega_{1}+\omega_{2}, \omega_{3}\right) \prod_{k=3}^{N-1} C_{k}(\omega) D(\omega) .
$$

We now consider, for every $l=0, \ldots, M-1$, the sets

$$
E_{2}+l \varepsilon^{(3)}=\{\varepsilon=(j+l, M-j, M-l, 0 \ldots, 0), j=0, \ldots, M-1\} .
$$

Then, obviously

$$
\begin{aligned}
\sum_{\varepsilon \in E_{2}+l \varepsilon^{(3)}} h(\omega+\vartheta \varepsilon) & =\sum_{\varepsilon \in E_{2}} h\left(\omega+l \varepsilon^{(3)} \vartheta+\varepsilon \vartheta\right) \\
& =f\left(\omega_{1}+\omega_{2}+l \vartheta\right)^{\alpha_{3}} f\left(\omega_{3}+(M-l) \vartheta\right)^{\alpha_{3}} \\
& \times A_{\alpha_{3}}\left(\omega_{1}+\omega_{2}+l \vartheta, \omega_{3}+(M-l) \vartheta\right) \prod_{k=3}^{N-1} C_{k}(\omega) D(\omega) .
\end{aligned}
$$

We now define the set

$$
E_{3}=\bigcup_{l=0}^{M-1}\left(E_{2}+l \varepsilon^{(3)}\right)
$$

which consists of the elements of $E$ with, at most, the first three coordinates different from zero and having sum equal to 0 . Obviously $\left|E_{3}\right|=M^{2}$. Then, using again Lemma 2 and the definition of $\alpha_{4}$, we get

$$
\begin{aligned}
\sum_{\varepsilon \in E_{3}} h(\omega+\vartheta \varepsilon) & =f\left(\omega_{1}+\omega_{2}+\omega_{3}\right)^{\alpha_{4}} f\left(\omega_{4}\right)^{\alpha_{4}} A_{\alpha_{4}}\left(\omega_{1}+\omega_{2}+\omega_{3}, \omega_{4}\right) \\
& \times \prod_{k=4}^{N-1} C_{k}(\omega) D(\omega) .
\end{aligned}
$$

At the $j$-th step we define recursively the set $E_{j}(j=2, \ldots, N)$ as

$$
E_{j}=\bigcup_{l=0}^{M-1}\left(E_{j-1}+l \varepsilon^{(j)}\right)
$$


Notice that $\left|E_{j}\right|=M^{j-1}$ and that $E_{j}$ consists of the elements of $E$ with, at most, the first $j$ coordinates different from zero and having sum equal to 0 . Furthermore,

$$
\begin{aligned}
\sum_{\varepsilon \in E_{j}} h(\omega+\vartheta \varepsilon) & =f\left(\omega_{1}+\cdots+\omega_{j}\right)^{\alpha_{j+1}} f\left(\omega_{j+1}\right)^{\alpha_{j+1}} A_{\alpha_{j+1}}\left(\omega_{1}+\cdots+\omega_{j}, \omega_{j+1}\right) \\
& \times \prod_{k=j+1}^{N-1} C_{k}(\omega) D(\omega) .
\end{aligned}
$$

At the last step (also using the definition of $\alpha_{N+1}$ ) we obtain

$$
\sum_{\varepsilon \in E_{N}} h(\omega+\vartheta \varepsilon)=f\left(\omega_{1}+\cdots+\omega_{N}\right)^{2 \alpha_{N+1}} B_{\alpha_{N+1}}\left(\omega_{1}+\cdots+\omega_{N}\right),
$$

where $E_{N}$ is the subgroup of the elements of $E$ whose sum is $0 \bmod M$. Now consider the elements of $E$ of the form

$$
\eta^{(j)}=(j, 0, \ldots, 0) \quad j=0, \ldots, M-1 .
$$

Then $E$ is the disjoint union of the cosets $E_{N}+\eta^{(j)}$. Hence, it follows from Lemma 3 that

$$
\begin{aligned}
\sum_{\varepsilon \in E} h(\omega+\vartheta \varepsilon) & =\sum_{j=0}^{M-1} \sum_{\varepsilon \in E_{N}+\eta^{(j)}} h(\omega+\vartheta \varepsilon) \\
& =\sum_{j=0}^{M-1} f\left(\omega_{1}+\cdots+\omega_{N}+j \vartheta\right)^{2 \alpha_{N+1}} B_{\alpha_{N+1}}\left(\omega_{1}+\cdots+\omega_{N}+j \vartheta\right) \\
& =1 .
\end{aligned}
$$

Remark 4. In the case $N=1$, Conditions (41) must simply be replaced by the condition $2 \alpha_{2}=\alpha_{1}$.

\section{Regularity}

In this section we show that, for fixed exponents $a_{k}$ and $b_{k}$ in $m_{0}$ (38) it is possible to choose $\alpha_{k}$ and $\beta_{k}$ in $\widetilde{m}_{0}$ (39) in such a way that $\widehat{\widetilde{\varphi}}$ has arbitrarily fast (polynomial) decay. At the same time we will show that (24) can be satisfied. To this end we will use the regularity exponent of a filter, a notion thoroughly investigated in the literature (see e.g., [5], [8], [10], [26] in the case $M=2$ and [1], [14], [16], [23], [24], [25] in the general case).

Let $p(t)$ be a trigonometric polynomial such that $p(k \vartheta)=\delta_{k, 0}$ for $k=$ $0, \ldots, M-1$. Then, there exists a positive integer $L$ such that

$$
p(t)=\left(\frac{1+e^{-i t}+e^{-i 2 t}+\cdots+e^{-i(M-1) t}}{M}\right)^{L} P(t),
$$


where $P$ is a trigonometric polynomial such that $P(0)=1$ and $P(k \vartheta) \neq 0$ for $k \neq 0$. Set

$$
\begin{aligned}
& \mathcal{K}_{\ell}=\mathcal{K}_{\ell}(P)=\frac{1}{\ell} \log _{M}\left(\sup _{t} \prod_{h=1}^{\ell}\left|P\left(M^{-h} t\right)\right|\right), \\
& \mathcal{K}=\mathcal{K}(P)=\inf _{\ell} \mathcal{K}_{\ell}(P)=\lim _{\ell \rightarrow \infty} \mathcal{K}_{\ell}(P) .
\end{aligned}
$$

The number $\mathcal{K}(P)$ is called the regularity exponent of $P$. It is known (see e.g [8, p. 82]) that for every $\ell$ there exists a constant $C_{\ell}$ such that, for every $n \geq \ell$, one has

$$
\prod_{h=1}^{n}\left|P\left(M^{-h} t\right)\right| \leq C_{\ell}(1+|t|)^{\mathcal{K}_{\ell}}
$$

Therefore, we have $\left|\prod_{h=1}^{\infty} p\left(M^{-h} t\right)\right| \leq C_{\ell}(1+|t|)^{-L+\mathcal{K}_{\ell}}$, so that, depending on the value of $\mathcal{K}$, the infinite product belongs to various functional spaces. We shall use (42) to prove not only the validity of (24) but also the regularity of the dual scaling function.

We start recalling that, for

$$
\begin{aligned}
P_{S}(t) & =\sum_{|j|=M(s-1)+1}\left(\begin{array}{c}
M(s-1)+1 \\
j
\end{array}\right) g\left(\frac{j_{0}}{M(s-1)+1}, \ldots, \frac{j_{M-1}}{M(s-1)+1}\right) \\
& \times\left(\frac{\sin ^{2}\left(\frac{M t}{2}\right)}{M^{2} \sin ^{2}\left(\frac{t}{2}\right)}\right)^{j_{0}-s} \prod_{\ell=1}^{M-1}\left(\frac{\sin ^{2}\left(\frac{M t}{2}\right)}{M^{2} \sin ^{2}\left(\frac{t}{2}+\frac{\ell \pi}{M}\right)}\right)^{j_{\ell}},
\end{aligned}
$$

the following asymptotic estimates for $\mathcal{K}\left(P_{S}\right)$ are known. If $M=2$ then, as $s \rightarrow \infty, \mathcal{K}\left(P_{s}\right) \sim s \log _{2} 3$ [5], [26]. If $M=3$, then $\mathcal{K}\left(P_{s}\right) \sim s\left(-1+\log _{3} 16\right)$, [23, Theorems 7 and 9]. Finally, if $M>3$ then, for $s$ sufficiently large one has $\mathcal{K}\left(P_{s}\right)<s\left(1+(M-1) \log _{M}\left(\frac{M}{M-1}\right)\right)$, [24], [23, Theorem 7].

Thus we set

$$
\rho_{M}= \begin{cases}\log _{2} 3 & \text { if } M=2 \\ -1+\log _{3} 16 & \text { if } M=3 \\ 1+(M-1) \log _{M}\left(\frac{M}{M-1}\right) & \text { if } M>3\end{cases}
$$

Note that

$$
0<\rho_{M}<2 \text { for all } M \text {. }
$$

Theorem 3. Let $\tilde{m}_{0}$ be as in (39) with $r$ as in (40). Then, for every $\mu>0$, there exist a constant $C>0$, and exponents $\alpha_{k}$ and $\beta_{k}$ satisfying Conditions (41) of Theorem 2 such that, for every sufficiently large $n$, one has

$$
\prod_{h=1}^{n}\left|r\left(M^{-h} \omega\right)\right| \leq C \prod_{k=1}^{N}\left(1+\left|\omega_{k}\right|\right)^{\alpha_{k}-a_{k}-\mu} \times \prod_{k=2}^{N}\left(1+\left|\omega_{1}+\cdots+\omega_{k}\right|\right)^{\beta_{k}-b_{k}-\mu} .
$$


Proof. Let $P_{s}$ be as in (43) above. Then, remembering the explicit form of $A_{k}$ and using the Schwartz inequality we have, for every $k \leq N-1$,

$$
\left|A_{\alpha_{k+1}}\left(\omega_{1}+\cdots+\omega_{k}, \omega_{k+1}\right)\right| \leq P_{\alpha_{k+1}}^{1 / 2}\left(\omega_{1}+\cdots+\omega_{k}\right) P_{\alpha_{k+1}}^{1 / 2}\left(\omega_{k+1}\right) .
$$

Of course, $\left|B_{\alpha_{N+1}}\left(\omega_{1}+\cdots+\omega_{N}\right)\right|=P_{\alpha_{N+1}}\left(\omega_{1}+\cdots+\omega_{N}\right)$. Thus

$$
\begin{aligned}
\prod_{h=1}^{n}\left|r\left(M^{-h} \omega\right)\right| & \leq \prod_{k=1}^{N} \prod_{h=1}^{n} P_{\alpha_{k}}^{1 / 2}\left(M^{-h} \omega_{k}\right) \prod_{k=2}^{N-1} \prod_{h=1}^{n} P_{\alpha_{k+1}}^{1 / 2}\left(M^{-h}\left(\omega_{1}+\cdots+\omega_{k}\right)\right) \\
& \times \prod_{h=1}^{n} P_{\alpha_{N+1}}\left(M^{-h}\left(\omega_{1}+\cdots+\omega_{N}\right)\right) .
\end{aligned}
$$

Set $\mathcal{K}(\ell, s)=\mathcal{K}_{\ell}\left(P_{s}\right)$ and $\mathcal{K}(s)=\mathcal{K}\left(P_{s}\right)$. Then, for every $\ell$, if $n \geq \ell$ we get

$$
\begin{aligned}
& \prod_{h=1}^{n}\left|r\left(M^{-h} \omega\right)\right| \leq C_{\ell} \prod_{k=1}^{N}\left(1+\left|\omega_{k}\right|\right)^{\mathcal{K}\left(\ell, \alpha_{k}\right) / 2} \\
& \quad \times \prod_{k=2}^{N-1}\left(1+\left|\omega_{1}+\cdots+\omega_{k}\right|\right)^{\mathcal{K}\left(\ell, \alpha_{k+1}\right) / 2} \times\left(1+\left|\omega_{1}+\cdots+\omega_{N}\right|\right)^{\mathcal{K}\left(\ell, \alpha_{N+1}\right)} .
\end{aligned}
$$

Choose $\varepsilon$ such that $\rho_{M}(1+\varepsilon)<2$ (this is possible by (44)). Then, choose the $\alpha_{k}$ so large that $\mathcal{K}\left(\alpha_{k}\right) \leq \alpha_{k} \rho_{M}(1+\varepsilon / 2)$. For this choice of the $\alpha_{k}$ choose $\ell$ so large that $\mathcal{K}\left(\ell, \alpha_{k}\right) \leq \mathcal{K}\left(\alpha_{k}\right)+\varepsilon \alpha_{k} \rho_{M} / 2$. Then we get, from (45),

$$
\begin{aligned}
& \prod_{h=1}^{n}\left|r\left(M^{-h} \omega\right)\right| \leq C \prod_{k=1}^{N}\left(1+\left|\omega_{k}\right|\right)^{\alpha_{k} \rho_{M}(1+\varepsilon) / 2} \\
\times & \prod_{k=2}^{N-1}\left(1+\left|\omega_{1}+\cdots+\omega_{k}\right|\right)^{\alpha_{k+1} \rho_{M}(1+\varepsilon) / 2} \times\left(1+\left|\omega_{1}+\cdots+\omega_{N}\right|\right)^{\alpha_{N+1} \rho_{M}(1+\varepsilon)} .
\end{aligned}
$$

To complete the proof we have to show that there exist $\beta_{k}$ and arbitrarily large $\alpha_{k}$ which satisfy (41) and

$$
\begin{aligned}
\alpha_{k} \rho_{M} \frac{(1+\varepsilon)}{2} \leq \alpha_{k}-a_{k}-\mu, & 1 \leq k \leq N \\
\alpha_{k+1} \rho_{M} \frac{(1+\varepsilon)}{2} \leq \beta_{k}-b_{k}-\mu, & 2 \leq k \leq N-1 \\
2 \alpha_{N+1} \rho_{M} \frac{(1+\varepsilon)}{2} \leq \beta_{N}-b_{N}-\mu & k=N .
\end{aligned}
$$

Let $\alpha_{1}>0$ be such that $\alpha_{1}\left(1-\rho_{M} \frac{(1+\varepsilon)}{2}\right)>\mu+\max _{k}\left(a_{k}\right)$; this is possible by (44). Then every $\alpha_{k} \geq \alpha_{1}$ satisfies the first set of inequalities. By defining $\beta_{k}$ by means of (41), the second set of inequalities can be recast as

$$
\alpha_{k+1}\left(1-\rho_{M} \frac{(1+\varepsilon)}{2}\right) \geq M\left(\alpha_{k}-1\right)+1+b_{k}+\mu,
$$


which can be recursively satisfied in the range $\alpha_{k} \geq \alpha_{1}$. Analogously, we see that the last inequality can be satisfied for large values of $\alpha_{N+1}$.

Finally we get that $\tilde{\varphi}$ can have a Fourier transform with arbitrarily fast decay in the directions of $\Xi$.

Corollary 1. Let $\tilde{m}_{0}$ be as in (39) with $r$ as in (40). Moreover, let $\widehat{\widetilde{\varphi}}(\omega)=$ $\prod_{j=1}^{\infty} \tilde{m}_{0}\left(M^{-j} \omega\right)$. Then, for every $\mu>0$, there exist a constant $C>0$, and exponents $\alpha_{k}$ and $\beta_{k}$ satisfying Conditions (41) of Theorem 2 such that

$$
|\widehat{\widetilde{\varphi}}(\omega)| \leq C \prod_{k=1}^{N}\left(1+\left|\omega_{k}\right|\right)^{-\mu} \prod_{k=2}^{N}\left(1+\left|\omega_{1}+\cdots+\omega_{k}\right|\right)^{-\mu} .
$$

\section{References}

1. Bi, N., Debnath, L., Sun, Q.: Asymptotic behaviour of $M$-band scaling functions of Daubechies type. Z. Anal. Anwend. 17, 813-830 (1998)

2. deBoor, C., Höllig, K., Riemenschneider, S.: Box splines. New York: Springer 1993

3. Caglar, H., Akansu, N.: A generalized parametric PR-QF design technique based on Bernstein polynomial approximation. IEEE Trans. Signal Processing 41, 2314-2321 (1993)

4. Chui, C.K., Li, C.: A general framework of multivariate wavelets with duals. Appl. Comp. Harmonic. Anal. 1, 368-390 (1994)

5. Cohen, A., Conze, J.P., Régularité des bases d'ondelettes et mesures ergodiques. Rev. Mat. Iberaoamer. 8, 351-365 (1992)

6. Cohen, A., Daubechies, I.: Nonseparable bidimensional wavelet bases. Revista Mat. Iberoamer. 9, 51-137 (1993)

7. Cohen, A., Daubechies, I., Feauveau, J.C.: Biorthogonal bases of compactly supported wavelets. Comm. Pure Appl. Math. 44, 485-560 (1992)

8. Cohen, A., Ryan, R.D.: Wavelets and multiscale signal processing. London: Chapman and Hall 1995

9. Cohen, A., Schlenker, J.M. Compactly supported bidimensional wavelet base with hexagonal symmetry. Constr. Approx. 9, 209-236 (1993)

10. Daubechies, I.: Ten lectures on wavelets. Philadelphia: SIAM 1992

11. Deslauriers, G., Dubois, J., Dubuc, S.: Multidimensional iterative interpolation. Can. J. Math. 43, 297-312 (1991)

12. Dahlke, S., Gröchenig, K., Latour, V.: Biorthogonal box spline wavelet bases. In: Surface fittings and multiresolution methods, Le Méhauté, A., Rabut, C., Schumaker, L., eds., Nashville: Vanderbilt University Press 1997

13. He, W., Lai, M.J.: Construction of bivariate compactly supported box spline wavelets with arbitrarily high regularity. Appl. Comp. Harmonic. Anal. 6, 53-73 (1999)

14. Heller, P.N., Wells jr., R.O.: Sobolev regularity for rank $M$ wavelets. Technical Report 96-08, Computational Math. Lab. Rice Univ. 1996

15. Hernandez, E., Weiss, G.: A first course on wavelets. Boca Raton: CRC Press 1996

16. Ji, H., Riemenschneider, S., Shen, Z.: Multivariate compactly supported refinable functions, duals and biorthogonal wavelets. Stud. Appl. Math. 126, 173-204 (1999)

17. Lemarié-Rieusset, P.G.: Polynomes de Bernstein en théorie des ondelettes. C.R. Acad. Sci. Paris 319, Série I, 21-21 (1994)

18. Logar, A., Sturmfels, B.: Algorithms for the Quillen-Suslin theorem. J. Algebra 145, 231-239 (1992)

19. Lorentz, G.G.: Bernstein polynomials. New York: Chelsea Publ. Co. 1986 
20. Riemenschneider, S., Shen, Z.: Construction of compactly supported biorthogonal wavelets in $L^{2}\left(\mathbb{R}^{N}\right)$. In: Wavelets Application Signal and Image Processing VII, Michael A. Unser, Akram Aldroubi and Andrew F. Laine, eds., Proc. SPIE 3813, 1999, pp. 264-278

21. Riemenschneider, S., Shen, Z., Multidimensional interpolatory subdivision schemes. SIAM J. Numer. Anal. 34, 2357-2381 (1997)

22. Ron, A., Shen, Z.: Affine systems in $L^{2}\left(\mathbb{R}^{N}\right)$ II: dual systems. J. Fourier Anal. Appl. 3, 617-637 (1997)

23. Soardi, P.M.: Biorthogonal $M$-channel compactly supported wavelets. Constr. Approx. 16, 283-311 (2000)

24. Shi, X., Sun, Q.: A class of $M$-dilation scaling functions with regularity growing proportionally to filter support width. Proc. Amer. Math. Soc. 126, 3501-3506 (1998)

25. Sun, Q.: Sobolev index estimate and asymptotic regularity of $M$ band Daubechies scaling functions. Constr. Approx. 15, 441-465 (1999)

26. Volkmer, H.: On the regularity of wavelets. IEEE Trans. Information Theory $\mathbf{3 8}$, 872-876 (1992) 